\title{
Matchings Avoiding Partial Patterns
}

\author{
William Y. C. Chen ${ }^{1}$, Toufik Mansour ${ }^{2}$, Sherry H. F. Yan ${ }^{3}$ \\ ${ }^{1,3}$ Center for Combinatorics, LPMC \\ Nankai University, Tianjin 300071, P.R. China \\ ${ }^{2}$ Department of Mathematics, University of Haifa, Haifa 31905, Israel \\ ${ }^{1}$ chen@nankai.edu.cn, ${ }^{2}$ toufik@math.haifa.ac.il, ${ }^{3}$ huifangyan@eyou.com
}

Submitted: Apr 16, 2005; Accepted: Dec 6, 2006; Published: Dec 18, 2006

Mathematics Subject Classifications: 05A05, 05C30

\begin{abstract}
We show that matchings avoiding a certain partial pattern are counted by the 3Catalan numbers. We give a characterization of 12312-avoiding matchings in terms of restrictions on the corresponding oscillating tableaux. We also find a bijection between matchings avoiding both patterns 12312 and 121323 and Schröder paths without peaks at level one, which are counted by the super-Catalan numbers or the little Schröder numbers. A refinement of the super-Catalan numbers is derived by fixing the number of crossings in the matchings. In the sense of Wilf-equivalence, we use the method of generating trees to show that the patterns 12132, 12123, 12321, 12231, 12213 are all equivalent to the pattern 12312 .
\end{abstract}

\section{Introduction}

A matching on a set $[2 n]=\{1,2, \ldots, 2 n\}$ is a partition of $[2 n]$ in which every block contains exactly two elements, or equivalently, a graph on $[2 n]$ in which every vertex has degree one. There are many ways to represent a matching. It can be displayed by drawing the $2 n$ points on a horizontal line in the increasing order. This is called the linear representation of a matching [5]. An edge $e=(i, j)$ in a matching is always written in such a way that $i<j$, where the vertices $i$ and $j$ are called the initial point and the endpoint, respectively. We assume that every edge $(i, j)$ is drawn as an arc between the nodes $i$ and $j$ above the horizontal line. Let $e=(i, j)$ and $e^{\prime}=\left(i^{\prime}, j^{\prime}\right)$ be two edges of a matching $M$, we say that $e$ crosses $e^{\prime}$ if they intersect with each other, in other words, if $i<i^{\prime}<j<j^{\prime}$. In this case, the pair of edges $e$ and $e^{\prime}$ is called a crossing of the matching. Otherwise, $e$ and $e^{\prime}$ are said to be noncrossing. The set of matchings on $[2 n]$ is denoted by $\mathcal{M}_{n}$. 
In this paper, we also use the representation of a matching $M$ with $n$ edges by a sequence of length $2 n$ on the set $\{1,2, \ldots, n\}$ such that each element $i(1 \leq i \leq n)$ appears exactly twice, and the first occurrence of the element $i$ precedes that of $j$ if $i<j$. Such a representation is called the Davenport-Schinzel sequence [9, 24] or the canonical sequential form [21]. In fact, the canonical sequential form of a matching is the sequence obtained from its linear representation by labeling the endpoints in accordance with the order of the appearances of the initial points. For example, the matching in Figure 1 can be represented by 123123 in the canonical sequential form.

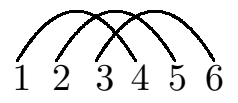

Figure 1: The matching 123123.

Given a sequence $a_{1} a_{2} \cdots a_{m}$ of integers, we define its pattern as a sequence obtained by replacing the minimum element (which may have repeated occurrences) by 1 , and replacing the second minimum element by 2, and so on. For example, the pattern of the sequence 322962538256 is 211641325134 . In this paper, we are mainly concerned with the partial pattern 12312 in the sense that it does not form a complete matching. In the terminology of canonical sequential form, we say that a matching $\pi$ avoids a pattern $\tau$, or $\pi$ is $\tau$-avoiding, if there is no subsequence of the pattern $\tau$ in $\pi$. The set of $\tau$-avoiding matchings on $[2 n]$ is denoted by $\mathcal{M}_{n}(\tau)$. Similarly, we use $M_{n}\left(\tau_{1}, \tau_{2}, \ldots, \tau_{k}\right)$ to denote the set of matchings on $[2 n]$ which avoid patterns $\tau_{1}, \tau_{2}, \ldots, \tau_{k}$. Pattern avoiding matchings have been studied by de Médicis and Viennot [25], de Sainte-Catherine [28], Gessel and Viennot [16], Gouyou-Beauchamps [18, 19], Stein [33], Touchard [36], and recently by Klazar [21, 22, 23], Chen, Deng, Du, Stanley and Yan [6].

The $k$-Catalan numbers, or generalized Catalan numbers are defined by

$$
C_{n, k}=\frac{1}{(k-1) n+1}\left(\begin{array}{c}
k n \\
n
\end{array}\right)
$$

for $n \geq 1$ (see [20]). For $k=2$, the 2-Catalan numbers are the usual Catalan numbers.

The main objective of this paper is to show that 12312-avoiding matchings on $[2 n]$ are counted by the 3-Catalan numbers, namely,

$$
\left|\mathcal{M}_{n}(12312)\right|=\frac{1}{2 n+1}\left(\begin{array}{c}
3 n \\
n
\end{array}\right) .
$$

We note that the following objects are also counted by the 3-Catalan numbers:

- complete ternary trees with $n$ internal vertices, or $3 n$ edges [26],

- even trees with $2 n$ edges $[4,12]$,

- noncrossing trees with $n$ edges $[13,26]$, 
- the set of lattice paths from $(0,0)$ to $(2 n, n)$ using steps $E=(1,0)$ and $N=(0,1)$ and never lying above the line $y=x / 2$ [20],

- dissections of a convex $(2 n+2)$-gon into $n$ quadrilaterals by drawing $n-1$ diagonals, no two of which intersect in its interior [20],

- two line arrays $\left(\begin{array}{c}\alpha \\ \beta\end{array}\right)$, where $\alpha=\left\{a_{1}, a_{2}, \ldots, a_{n}\right\}$ and $\beta=\left\{b_{1}, b_{2}, \ldots, b_{n}\right\}$ such that $1=b_{1}=a_{1} \leq b_{2} \leq a_{2} \ldots \leq b_{n} \leq a_{n}$ and $a_{i} \leq i[3]$.

The relations among ternary trees, even trees, and noncrossing trees have been studied by Chen [4], Feretic and Svrtan [14], Noy [15], and Panholzer and Prodinger [26]. Stanley discussed several of these families in [32, Problems 5.45 - 5.47].

By using generating functions, we derive a formula for the number of matchings in $\mathcal{M}_{n}(12312)$ having exactly $m$ crossings. We also show that the cardinality of $\mathcal{M}_{n-1}(12312$, 121323 ) is the $n$-th super-Catalan number or the little Schröder number for $n \geq 1$ (see [29, Sequence A001003]). By considering the number of matchings in $\mathcal{M}_{n-1}(12312,121323)$ having exactly $m$ crossings we obtain a closed expression as a refinement of the superCatalan numbers. The $n$-th super-Catalan number also equals the number of Schröder paths of semilength $n-1$ (i.e. lattice paths from $(0,0)$ to $(2 n-2,0)$, with steps $H=(2,0)$, $U=(1,1)$, and $D=(1,-1)$ and not going below the $x$-axis) without peaks at level one, as well as certain Dyck paths (see [29, Sequence A001003] and references therein). We find a bijection between Schröder paths of semilength $n$ without peaks at level one and matchings on [2n] avoiding both patterns 12312 and 121323.

Following the approach of Chen, Deng, Du, Stanley and Yan [6], we use oscillating tableaux to study 12312-avoiding matchings. The notion of oscillating tableaux first appeared in the study of the decomposition formula for powers of defining representations of the complex symplectic groups by Berele [2]. We will use the bijection between matchings and oscillating tableaux originally due to Stanley [32, Exercise 7.24] and later extended by Sundaram $[34,35]$ (see also $[10,27]$ ). Recall that an oscillating tableau of shape $\lambda$ is a sequence of Young diagrams (or partitions) $\emptyset=\lambda^{0}, \lambda^{1}, \ldots \lambda^{k-1}, \lambda^{k}=\lambda$ such that the diagram $\lambda^{i}$ is obtained from $\lambda^{i-1}$ by either adding one square or removing one square. An oscillating tableau can be equivalently formulated as a sequence of standard Young tableaux (often abbreviated as SYT). The number $k$ in the above definition is called the length of the oscillating tableau. We denote by $T_{k}^{\lambda}$ the set of oscillating tableaux of shape $\lambda$ and length $k$.

For 12312-avoiding matchings we obtain the corresponding oscillating tableaux and closed lattice walks. We further provide a one-to-one correspondence between the set of closed lattice walks and the set of lattice paths from $(0,0)$ to $(2 n, n)$ using steps $E=(1,0)$ and $N=(0,1)$ without crossing the line $y=x / 2$, see [17]. From this perspective, we see that $\mathcal{M}_{n}(12312)$ is counted by the 3-Catalan number $C_{n, 3}$.

In addition to the pattern 12312, we find other patterns that are equivalent to 12312 in the sense of Wilf-equivalence. To be more specific, we show that for any pattern 
$\tau \in\{12312,12132,12123,12321,12231,12213\}$, we have $\left|M_{n}(\tau)\right|=C_{n, 3}$. We use the technique of generating trees to reach this conclusion. A generating tree is a rooted tree in which each vertex is associated with a label, and the labels of the children of any vertex are determined by certain succession rules. The idea of generating trees was introduced by Chung, Graham, Hoggat and Kleiman [8] in their study of Baxter permutations, and it has become an efficient method for many enumeration problems, see, for example, Barcucci, del Lungo, Pergola, and Pinzani [1], Stankova [30, 31], and West [37, 38].

\section{Matchings and Ternary Trees}

In this section, we use the linear representation of a matching as described in the introduction. Our goal is to show that the cardinality of $\mathcal{M}_{n}(12312)$ is equal to $C_{n, 3}$. The definition of a 12312-avoiding matching $M$ implies that there are no two crossing edges $e=(i, j)$ and $e^{\prime}=\left(i^{\prime}, j^{\prime}\right)$ with $i<i^{\prime}<j<j^{\prime}$ such that there is an initial point of a third edge between the nodes $i^{\prime}$ and $j$. Our first approach is to decompose a 12312-avoiding matching into smaller 12312-avoiding matchings. For notational convenience, we denote by $E_{j}$ the edge $(i, j)$ with $i<j$.

Lemma 2.1 Let $M$ be a 12312-avoiding matching on $[2 n]$ with $E_{2 n}=(j, 2 n)$. Suppose that there are $m$ edges crossing $E_{2 n}$. Let $v_{0}=0$ and $v_{s}$ be the rightmost end point of an edge crossing $E_{j+m+1-s}$. If no such an edge exists, we define $v_{s}$ as the initial point of $E_{j+m+1-s}$. Then $M$ can be decomposed into $m+2$ smaller 12312 -avoiding matchings $\theta_{1}, \theta_{2}, \ldots, \theta_{m}, \alpha, \beta$ such that

- $\theta_{s}$ is the induced subgraph of $M$ on the nodes $v_{s-1}+1, v_{s-1}+2, \ldots, v_{s}$, $j+m+1-s$ for $s \geq 1$;

- $\alpha$ is the induced subgraph of $M$ on the nodes $v_{m}+1, v_{m}+2, \ldots, j-1$ when $v_{m}+1<j$; otherwise it is empty;

- $\beta$ is the induced subgraph of $M$ on the nodes $j+m+1, j+m+2, \ldots, 2 n-1$ when $j+m+1<2 n$; otherwise it is empty.

Proof. If there is no edge crossing $(j, 2 n)$, then it is clear that $M$ can be decomposed into two smaller matchings $\alpha$ and $\beta$ such that $\alpha$ is a 12312-avoiding matching on the nodes $1,2, \ldots, j-1$ when $j>1$ and $\beta$ is a 12312 -avoiding matching on the nodes $j+1, j+$ $2, \ldots, 2 n-1$ when $j+1<2 n$.

If there is at least one edge crossing $(j, 2 n)$, then let $j+m$ be the rightmost end point of an edge crossing $(j, 2 n)$. Thus the nodes $j+1, j+2, \ldots, j+m-1$ cannot be the initial points, which implies that $E_{j+1}, E_{j+2}, \ldots, E_{j+m}$ are the $m$ edges crossing $E_{2 n}$. Therefore, the induced subgraph on the nodes $j+m+1, j+m+2, \ldots, 2 n-1$ is a 12312-avoiding matching when $j+m+1<2 n$, which we denote by $\beta$. 
Since $M$ is a 12312 -avoiding matching, we have $v_{0}<v_{1}<\ldots<v_{m}$. Note that there is no initial point between the initial point of $E_{j+m+1-k}$ and the node $v_{k}$ for $1 \leq k \leq m$. It follows that the induced subgraph on the nodes $v_{s-1}+1, \ldots, v_{s}, j+m+1-s$ is a 12312 -avoiding matching for $1 \leq s \leq m$. Let us denote this matching by $\theta_{s}$. Hence the induced subgraph on the nodes $v_{m}+1, v_{m}+2, \ldots, j-1$ is a 12312 -avoiding matching when $v_{m}+1<j$, which we denote by $\alpha$. So we can decompose the matching $M$ into $m+2$ smaller 12312-avoiding matchings.

Figure 2 is an illustration of Lemma 2.1.

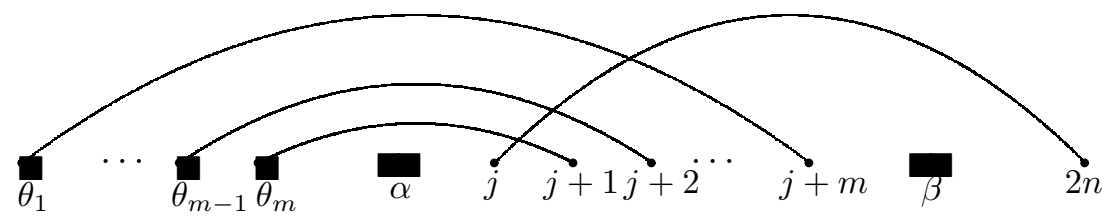

Figure 2: The decomposition

As a corollary of Lemma 2.1, we obtain a formula for the number of 12312-avoiding matchings on $[2 n]$ with exactly $m$ crossings.

Theorem 2.2 The number of 12312-avoiding matchings on [2n] with exactly $m$ crossings is given by

$$
\frac{1}{n}\left(\begin{array}{c}
n-1+m \\
n-1
\end{array}\right)\left(\begin{array}{c}
2 n-m \\
n+1
\end{array}\right)
$$

Proof. Let

$$
G(x, y)=\sum_{n \geq 0} \sum_{\theta \in M_{n}(12312)} x^{n} y^{\chi(\theta)}
$$

where $\chi(\theta)$ is the number of crossings of $\theta$. Let

$$
B(x, y)=\sum_{n \geq 1} \sum_{\theta} x^{n} y^{\chi(\theta)}
$$

where the second summation ranges over matchings $\theta_{s}$ as in Lemma 2.1. It follows from Lemma 2.1 that the ordinary generating function for the number of 12312-avoiding matchings with exactly $m$ edges crossing $E_{2 n}$ is given by $x y^{m} G^{2}(x, y) B^{m}(x, y)$. Summing over all the possibilities for $m \geq 0$ we arrive at

$$
G(x, y)=1+\frac{x G^{2}(x, y)}{1-y B(x, y)}
$$

Applying Lemma 2.1 for matchings of the form $\theta_{s}$, it follows that that the ordinary generating function for the number of 12312-avoiding matchings $\theta_{s}$ with exactly $k$ edges crossing 
$E_{j+m+1-s}$ is given by $x y^{k} G(x, y) B^{k}(x, y)$. Therefore, summing over all the possibilities for $k \geq 0$ we get

$$
B(x, y)=\frac{x G(x, y)}{1-y B(x, y)} .
$$

Combining (2.1) and (2.2) we obtain

$$
B(x, y)=\frac{G(x, y)-1}{G(x, y)} .
$$

It follows from (2.1) and (2.3) that $G(x, y)$ satisfies the following recurrence relation

$$
x G(x, y)^{3}+G(x, y)-G(x, y)^{2}+y(G(x, y)-1)^{2}=0 .
$$

Substituting $x y$ by $x$ and $y+1$ by $y$, we get

$$
G(x y, y+1)=1+y\left(x G^{3}(x y, y+1)+(G(x y, y+1)-1)^{2}\right) .
$$

Using the Lagrange inversion formula we obtain

$$
G(x y, y+1)=1+\sum_{i \geq 1} \frac{1}{i} \sum_{j=0}^{i}\left(\begin{array}{l}
i \\
j
\end{array}\right)\left(\begin{array}{c}
3 j \\
i+1+j
\end{array}\right) x^{j} y^{i}
$$

which implies that

$$
G(x, y)=1+\sum_{i \geq 1} \frac{1}{i} \sum_{j=0}^{i}\left(\begin{array}{l}
i \\
j
\end{array}\right)\left(\begin{array}{c}
3 j \\
i+1+j
\end{array}\right) x^{j}(y-1)^{i-j} .
$$

Then $\left[x^{n} y^{m}\right] G(x, y)$ gives the number of 12312-avoiding matchings on $[2 n]$ with exactly $m$ crossings. Applying an identity given in [7], we get

$$
\sum_{i=n}^{2 n-1}(-1)^{i-n-m}\left(\begin{array}{c}
3 n \\
n+1+i
\end{array}\right)\left(\begin{array}{c}
i-1 \\
n-1
\end{array}\right)\left(\begin{array}{c}
i-n \\
m
\end{array}\right)=\left(\begin{array}{c}
n-1+m \\
n-1
\end{array}\right)\left(\begin{array}{c}
2 n-m \\
n+1
\end{array}\right) .
$$

This completes the proof.

Setting $y=1$ in (2.6), we obtain the following conclusion.

Theorem 2.3 The number of 12312-avoiding matchings on [2n] equals the 3-Catalan number $C_{n, 3}$.

In principle, we may use the recursive structure of 12312-avoiding matchings to construct a bijection with ternary trees. However, as we will see it is more convenient to construct a direct bijection between 12312-avoiding matchings and oscillating tableaux. Then we can establish a correspondence between oscillating tableaux and lattice paths which are counted by the 3 -Catalan numbers. 


\section{$3 \mathcal{M}_{n}(12312,121323)$ and Schröder Paths}

In this section, we show that matchings avoiding both patterns 12312 and 121323 are in one-to-one correspondence with Schröder paths without peaks at level one. Such paths are counted by the super-Catalan numbers or the little Schröder numbers. We need a refinement of Lemma 2.1.

Lemma 3.1 Let $M$ be a matching on $[2 n]$ with $E_{2 n}=(j, 2 n)$ that avoids both patterns 12312 and 121323. Suppose that there are $m$ edges crossing $E_{2 n}$. Let $v_{0}=0, v_{m+1}=j$, and $v_{s}$ be the initial point of the edge $E_{j+m+1-s}$. Then $M$ can be decomposed into $m+2$ smaller matchings $\theta_{1}, \ldots, \theta_{m+1}, \beta$ avoiding both patterns 12312 and 121323 such that

1. $\theta_{s}$ is the induced subgraph of $M$ on the nodes $v_{s-1}+1, v_{s-1}+2, \ldots, v_{s}-1$ when $v_{s-1}+1<v_{s}$; otherwise it is empty;

2. $\beta$ is the induced subgraph of $M$ on the nodes $j+m+1, j+m+2, \ldots, 2 n-1$ when $j+m+1<2 n$; otherwise it is empty.

Figure 3 is an illustration of Lemma 3.1.

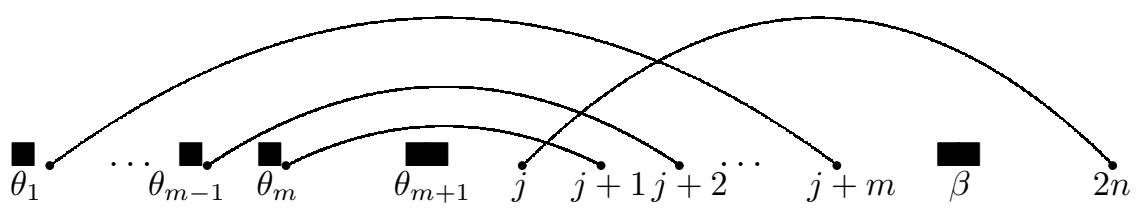

Figure 3: The refined decomposition

Let

$$
F(x)=\sum_{n \geq 0} f_{n} x^{n}
$$

be the ordinary generating function of the number of matchings on [2n] which avoid both patterns 12312 and 121323 . Lemma 3.1 leads to the following recurrence relation

$$
F(x)=1+\frac{x F^{2}(x)}{1-x F(x)}
$$

So we have

$$
F(x)=\frac{1+x-\sqrt{1-6 x+x^{2}}}{4 x}=1+\sum_{n \geq 1} \frac{1}{n} \sum_{j=1}^{n} 2^{j-1}\left(\begin{array}{c}
n \\
j
\end{array}\right)\left(\begin{array}{c}
n \\
j-1
\end{array}\right) x^{n} .
$$

Now we see that for $n \geq 1, f_{n-1}$ equals the $n$-th super-Catalan number which counts Schröder paths of semilength $n-1$ without peaks at level one. 
We proceed to give a bijection $\phi$ between the set of Schröder paths of semilength $n$ without peaks at level one and the set of matchings on $[2 n]$ which avoid both patterns 12312 and 121323. Note that any nonempty Schröder path $P$ has the following unique decomposition:

$$
P=H P^{\prime} \text { or } P=U P^{\prime} D P^{\prime \prime},
$$

where $P^{\prime}$ and $P^{\prime \prime}$ are possibly empty Schröder paths. This is called the first return decomposition by Deutsch [11].

Given a Schröder path $P$ of semilength $n$ without peaks at level one, if it is empty, then $\phi(P)$ is the empty matching. Otherwise, we may decompose it by using the first return decomposition. Moreover, we may use this decomposition recursively to get a matching $\phi(P)$ on $[2 n]$ avoiding both patterns 12312 and 121323. We have two cases.

(1) If $P=H P^{\prime}$, we have the structure as shown in Figure 4.

$$
\phi(P)=\overbrace{\phi\left(P^{\prime}\right)}
$$

Figure 4: Case 1

(2) If $P=U P^{\prime} D P^{\prime \prime}$ and $P^{\prime}=P_{1} U D P_{2} U D \ldots P_{k} U D P_{k+1}$, where for any $1 \leq i \leq k+1$, $P_{i}$ is a Schröder path without peaks at level one, then we have the structure as shown in Figure 5.

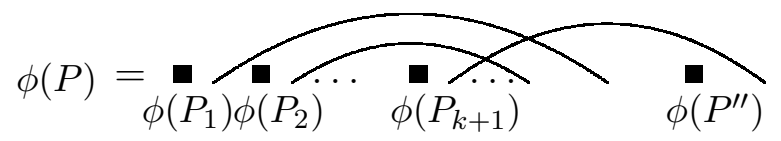

Figure 5: Case 2

Conversely, given a matching $M$ on [2n] which avoids both patterns 12312 and 121323 , we can construct a Schröder path $P$ of semilength $n$ without peaks at level one. Suppose that $M$ can be decomposed into smaller matchings $\theta_{1}, \ldots, \theta_{k+1}, \beta$ avoiding both patterns 12312 and 121323 as described in Lemma 3.1. If $k=0$ and $\theta_{1}=\emptyset$, then we have

$$
\phi^{-1}(M)=H \phi^{-1}(\beta)
$$

Otherwise, we get

$$
\phi^{-1}(M)=U \phi^{-1}\left(\theta_{1}\right) U D \phi^{-1}\left(\theta_{2}\right) U D \ldots \phi^{-1}\left(\theta_{k}\right) U D \phi^{-1}\left(\theta_{k+1}\right) D \phi^{-1}(\beta),
$$

which is a Schröder path of semilength $n$ without peaks at level one. Thus, we have obtained the desired bijection. 


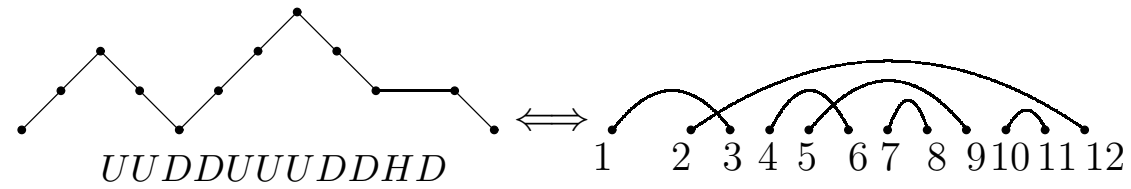

Figure 6: The bijection $\phi$

Example 3.2 As illustrated in Figure 6, the Schröder path UUDDUUUDDHD corresponds to the matching $\{(1,3),(2,12),(4,6),(5,9),(7,8),(10,11)\}$.

In view of the bijection $\phi$, we see that a peak in a Schröder path corresponds to a crossing of the corresponding matching. Let us use $\mathcal{M}_{n, m}(12312,121323)$ to denote the set of the matchings in $\mathcal{M}_{n}(12312,121323)$ with exactly $m$ crossings. We have the following formula which can be regarded as a refinement of the super-Catalan numbers, or the little Schröder numbers.

Theorem 3.3 For $n, m \geq 0$, we have

$$
\left|\mathcal{M}_{n, m}(12312,121323)\right|=\frac{1}{n}\left(\begin{array}{c}
n \\
m
\end{array}\right)\left(\begin{array}{c}
2 n-m \\
n+1
\end{array}\right) .
$$

Proof. It is well known that a Schröder path of semilength $n$ can be obtained from a Dyck path of semilength $n$ by turning some peaks of the Dyck path into $H$ steps. A peak is called a low peak if it is at level one; otherwise, it is called a high peak. It has been shown by Deutsch [11] that the number of Dyck paths of semilength $n$ with exactly $k$ high peaks is given by the Narayana number

$$
N(n, k)=\frac{1}{n}\left(\begin{array}{l}
n \\
k
\end{array}\right)\left(\begin{array}{c}
n \\
k+1
\end{array}\right) .
$$

Thus the number of Schröder paths of semilength $n$ that contain exactly $m$ high peaks but no peaks at level one equals

$$
\begin{aligned}
& \sum_{k=0}^{n-1} \frac{1}{n}\left(\begin{array}{l}
n \\
k
\end{array}\right)\left(\begin{array}{c}
n \\
k+1
\end{array}\right)\left(\begin{array}{c}
k \\
m
\end{array}\right) \\
& =\sum_{k=0}^{n-1} \frac{1}{n}\left(\begin{array}{l}
n \\
m
\end{array}\right)\left(\begin{array}{c}
n-m \\
k-m
\end{array}\right)\left(\begin{array}{c}
n \\
k+1
\end{array}\right) \\
& =\sum_{k=1}^{n} \frac{1}{n}\left(\begin{array}{c}
n \\
m
\end{array}\right)\left(\begin{array}{c}
n-m \\
n-k+1
\end{array}\right)\left(\begin{array}{l}
n \\
k
\end{array}\right) \\
& =\frac{1}{n}\left(\begin{array}{l}
n \\
m
\end{array}\right)\left(\begin{array}{c}
2 n-m \\
n+1
\end{array}\right) .
\end{aligned}
$$

This completes the proof. 


\section{Matchings and Oscillating Tableaux}

In this section, we apply Stanley's bijection between oscillating tableaux and matchings to derive a characterization of the oscillating tableaux for 12312-avoiding matchings. From the oscillating tableaux, we may construct closed lattice walks and lattice paths that are counted by the 3 -Catalan numbers.

Let us review the bijection of Stanley. Given an oscillating tableau $\emptyset=\lambda^{0}, \lambda^{1}, \ldots$, $\lambda^{2 n-1}, \lambda^{2 n}=\emptyset$, we may recursively define a sequence $\left(\pi_{0}, T_{0}\right),\left(\pi_{1}, T_{1}\right), \ldots,\left(\pi_{2 n}, T_{2 n}\right)$, where $\pi_{i}$ is a matching and $T_{i}$ is a standard Young tableau (SYT). Let $\pi_{0}$ be the empty matching and $T_{0}$ be the empty SYT. The tableau $T_{i}$ is obtained from $T_{i-1}$ and the matching $\pi_{i}$ is obtained from $\pi_{i-1}$ by the following rules:

1. If $\lambda^{i} \supset \lambda^{i-1}$, then $\pi_{i}=\pi_{i+1}$ and $T_{i}$ is obtained from $T_{i-1}$ by adding the entry $i$ in the square $\lambda^{i} \backslash \lambda^{i-1}$.

2. If $\lambda^{i} \subset \lambda^{i-1}$, then let $T_{i}$ be the unique SYT of shape $\lambda^{i}$ such that $T_{i-1}$ is obtained from $T_{i}$ by row-inserting some number $j$ by the RSK (Robinson-Schensted-Knuth) algorithm. In this case, let $\pi_{i}=\pi_{i-1} \cup(j, i)$.

If the entry $i$ is added to $T_{i-1}$ to obtain $T_{i}$, then we say that $i$ is added at step $i$. If $i$ is removed from $T_{j-1}$ to obtain $T_{j}$, then we say that $i$ leaves at step $j$. In this bijection, $(i, j)$ is an edge of the corresponding matching if and only if $i$ is added at step $i$ and leaves at step $j$.

Example 4.1 For the oscillating tableau

$$
\emptyset,(1),(2),(2,1),(1,1),(1), \emptyset
$$

we get the following sequence of SYTs:

$$
\begin{array}{lllllll}
\emptyset & 1 & 12 & 12 & 1 & 3 & \emptyset \\
& & & 3 & 3
\end{array}
$$

and the corresponding matching $\{(1,5),(2,4),(3,6)\}$.

The following theorem gives a characterization of oscillating tableaux corresponding to 12312-avoiding matchings.

Theorem 4.2 There exists a bijection $\rho$ between the set of 12312-avoiding matchings on $[2 n]$ and the set of oscillating tableaux $T_{2 n}^{\emptyset}$, in which each partition is of shape $(k)$ or $(k, 1)$ such that a partition $(k, 1)$ is not followed immediately by the partition $(k+1,1)$.

Proof. Let $M$ be a 12312-avoiding matching. By definition, there do not exist three edges $\left(i_{1}, j_{1}\right),\left(i_{2}, j_{2}\right)$ and $\left(i_{3}, j_{3}\right)$ such that $i_{1}<i_{2}<i_{3}<j_{1}<j_{2}$. Suppose that under 
Stanley's bijection, the sequence of SYTs corresponding to $M$ is $T_{0}, T_{1}, \ldots, T_{2 n}$ and the corresponding oscillating tableau is $\lambda^{0}, \lambda^{1}, \lambda^{2}, \ldots, \lambda^{2 n}$.

If $\lambda^{p-1}$ is of shape $(k)$ for some $1 \leq p \leq 2 n$, then it is possible that $\lambda^{p-1} \subset \lambda^{p}$. We claim that all the entries in $T_{p-1}$ must leave the tableau in deceasing order. Note that the entries in the first row of a SYT are strictly increasing. Suppose that $i_{2}$ is to the right of $i_{1}$ in $T_{p-1}$ and $i_{1}$ leaves the tableau before $i_{2}$. Assume that $i_{1}$ leaves at step $j_{1}$, namely, there exists an entry $h \geq p$ in the first row of $T_{j_{1}}$ such that $T_{j_{1}-1}$ is obtained from $T_{j_{1}}$ by inserting $i_{1}$. According to the RSK algorithm, the insertion of $i_{1}$ pushes $h$ up and $i_{1}$ takes the place of $h$. Hence in $T_{j_{1}}, h$ is to the left of $i_{2}$ in the first row, which contradicts the fact that $T_{j_{1}}$ is a SYT. So there do not exist two crossing edges $\left(i_{1}, j_{1}\right)$ and $\left(i_{2}, j_{2}\right)$ such that $i_{1}<i_{2}<p<j_{1}<j_{2}$. Hence $T_{p}$ can be obtained from $T_{p-1}$ by adding an entry $p$.

Suppose that $\lambda^{i-1}$ is of shape $(k)$ and $\lambda^{i}$ is of shape $(k, 1)$. According to the bijection $\rho, i$ is added to $\lambda^{i} \backslash \lambda^{i-1}$ to obtain $T_{i}$. It follows that the entry $i$ can only leave the SYT when it is moved to the first row. Suppose that $i$ is moved to the first row in $T_{j_{1}}$. Then there exists a unique entry $j$ such that $T_{j_{1}-1}$ is obtained from $T_{j_{1}}$ by row-inserting the entry $j$ by the RSK algorithm. Hence $j<i$ and $j$ leaves before $i$. Suppose that $i$ leaves the tableau at step $i_{1}$ with $j<i<j_{1}<i_{1}$, which implies that $\left(j, j_{1}\right)$ and $\left(i, i_{1}\right)$ are two crossing edges of the matching $M$. For any $i+1 \leq p \leq j_{1}$, we have $\lambda^{p} \subset \lambda^{p-1}$. Otherwise, $T_{p}$ is obtained from $T_{p-1}$ by inserting the entry $p$ in $\lambda^{p} \backslash \lambda^{p-1}$. It is clear that $p$ is the initial point of an edge of $M$. Let $\left(p, p_{1}\right)$ be an edge of $M$. Then $\left(j, j_{1}\right),\left(i, i_{1}\right)$ and $\left(p, p_{1}\right)$ are three edges of $M$ such that $j<i<p<j_{1}<i_{1}$, which contradicts the fact that $M$ is a 12312 -avoiding matching. Furthermore, $\lambda^{j_{1}}$ is of shape $(h)$ for some integer $h$. Therefore, if $\lambda^{p-1}$ is of shape $(k, 1)$, then no square is added to obtain $\lambda^{p}$ for any $1 \leq p \leq 2 n$. This completes the proof.

Given a matching $\pi \in \mathcal{M}_{n}(12312)$, we can define a closed lattice walk

$$
\left(x_{0}, y_{0}\right)=(0,0),\left(x_{1}, y_{1}\right),\left(x_{2}, y_{2}\right), \ldots,\left(x_{2 n}, y_{2 n}\right)=(0,0)
$$

such that $x_{i} \geq y_{i}$, where $x_{i}$ (resp. $y_{i}$ ) is the number of squares in the first (resp. second) row of the partition $\lambda^{i}$ of the corresponding oscillating tableau. Because of Theorem 4.2, if $\left(x_{i+1}, y_{i+1}\right)-\left(x_{i}, y_{i}\right)=(0,1)$, then the size of the next partition does not increase. Thus we have the following corollary.

Corollary 4.3 There is a one-to-one correspondence between 12312-avoiding matchings on $[2 n]$ and closed lattice walks of length $2 n$ in the $(x, y)$ plane from the origin to itself consisting of the steps $E=(1,0), W=(-1,0), N=(0,1)$ and $S=(0,-1)$ such that a step $N$ is followed immediately by some consecutive $W$ steps and one $S$ step and no step crosses the line $y=x$.

Example 4.4 The closed lattice walk in the $(x, y)$ plain corresponding to the matching $\{(1,5),(2,4),(3,6)\}$ is

\section{EENWSW}

starting from the origin and ending with the origin as well. 
Denote by $P_{n}$ the set of lattice paths from $(0,0)$ to $(2 n, n)$ consisting of steps $E=(1,0)$ and $N=(0,1)$ that never cross the line $y=x / 2$. Hilton and Pedersen [20] showed that the cardinality of $P_{n}$ is equal to $C_{n, 3}$. Let us use $L_{n}$ to denote the set of closed lattice walks specified in the above corollary.

We now give a one-to-one correspondence between the set $L_{n}$ and the set $P_{n}$. Given a closed lattice walk $p \in L_{n}$, we define a path $\tau(p)$ derived from $p$ by the following rule:

$$
\begin{aligned}
& E \rightarrow E E, \\
& W \rightarrow N, \\
& N \rightarrow E N, \\
& S \rightarrow E .
\end{aligned}
$$

Denote by $|p|_{E}$ the number of $E$ steps in the path $p$. We may similarly define $|p|_{W},|p|_{N}$ and $|p|_{S}$. Since $p$ is a lattice path going from the origin to itself with $2 n$ steps, we have $|p|_{E}=|p|_{W},|p|_{N}=|p|_{S}$ and $|p|_{E}+|p|_{N}=n$. From the map $\tau$, we have

$$
|\tau(p)|_{E}=2|p|_{E}+|p|_{N}+|p|_{S}=2 n
$$

and

$$
|\tau(p)|_{N}=|p|_{N}+|p|_{W}=n .
$$

Hence $\tau(p)$ goes from $(0,0)$ to $(2 n, n)$. We claim that $\tau(p)$ would never cross the line $y=x / 2$. Otherwise, let $\tau(p)=p_{1} p_{2} \ldots p_{3 n}$ such that $p_{k}=N$ is the first step going above the line $y=x / 2$. Let $q=p_{1} p_{2} \ldots p_{k}$ and $\tau\left(p^{\prime}\right)=q$. We have

$$
|q|_{E}=2\left|p^{\prime}\right|_{E}+\left|p^{\prime}\right|_{N}+\left|p^{\prime}\right|_{S}<2|q|_{N}=2\left|p^{\prime}\right|_{N}+2\left|p^{\prime}\right|_{W},
$$

which implies that either $\left|p^{\prime}\right|_{E}-\left|p^{\prime}\right|_{W}<\left|p^{\prime}\right|_{N}-\left|p^{\prime}\right|_{S}$ or $\left|p^{\prime}\right|_{E}<\left|p^{\prime}\right|_{W}$. This contradicts the fact that $p$ never goes above the line $y=x$. Thus we have $\tau(p) \in P_{n}$.

Conversely, given a lattice path $p \in P_{n}$, let $E_{i}$ be its $i$-th $E$ step from left to right. If $E_{2 k-1}$ and $E_{2 k}$ are consecutive steps in $p$, then $E_{2 k-1}$ together with $E_{2 k}$ corresponds to one $E$ step. Otherwise, $E_{2 k}$ corresponds to one $S$ step and $E_{2 k-1}$ together with its next step $N$ corresponds to one $N$ step. For the remaining $N$ steps, each $N$ step corresponds to one $W$ step. Suppose that we have obtained a path $p^{\prime}$. Then each $N$ step in $p^{\prime}$ is followed by some $W$ steps and one $S$ step and we have $\left|p^{\prime}\right|_{N}=\left|p^{\prime}\right|_{S}$. Moreover, we have the relations $|p|_{E}=2\left|p^{\prime}\right|_{E}+\left|p^{\prime}\right|_{N}+\left|p^{\prime}\right|_{S}=2 n$ and $|p|_{N}=\left|p^{\prime}\right|_{N}+\left|p^{\prime}\right|_{W}=n$. It follows that $\left|p^{\prime}\right|_{E}+\left|p^{\prime}\right|_{N}=n$ and $\left|p^{\prime}\right|_{E}=\left|p^{\prime}\right|_{W}$. Therefore, $p^{\prime}$ is a path from the origin to itself with $2 n$ steps. We claim that $p^{\prime}$ is a path never crossing the line $y=x$. Otherwise, let $p^{\prime}=p_{1} p_{2} \ldots p_{2 n}, q=p_{1} p_{2} \ldots p_{k}$ and $\tau^{-1}(w)=q$. From the map we have

$$
|w|_{E}=2|q|_{E}+|q|_{N}+|q|_{S} \geq 2|w|_{N}=2|q|_{N}+2|q|_{W}
$$

and either $|q|_{N}=|q|_{S}$ or $|q|_{N}-|q|_{S}=1$. Thus we have the inequality $|q|_{E}-|q|_{W} \geq$ $|q|_{N}-|q|_{S}$, which implies that $p^{\prime}$ is a path never crossing the line $y=x$. It follows that $p^{\prime} \in L_{n}$. Thus we have reached the following conclusion. 
Theorem 4.5 The map $\tau$ is a bijection between $L_{n}$ and $P_{n}$. Moreover, we have

$$
\left|\mathcal{M}_{n}(12312)\right|=\left|L_{n}\right|=\left|P_{n}\right|=\frac{1}{2 n+1}\left(\begin{array}{c}
3 n \\
n
\end{array}\right) .
$$

Example 4.6 For $n=2$, we have

$$
\begin{array}{cccc}
L_{2}: & E E W W, & E N S W, & E W E W, \\
& \downarrow & \downarrow & \downarrow \\
P_{2}: & E E E E N N, & E E E N E N, & E E N E E N .
\end{array}
$$

\section{$5 \quad$ Matchings and Generating Trees}

In this section, we use the methodology of generating trees to count matchings avoiding partial patterns. A generating tree is an infinite rooted tree which is essentially a process to generate labels from a single label of the root by successively applying certain rules. Formally speaking, a generating tree consists of the label of the root and the succession rules.

To make this paper self-contained, we use an example to explain the idea of generating trees. Let $S_{n}(\tau)$ be the set of $\tau$-avoiding permutations with $n$ elements. West [37] showed that $\left|S_{n}(123)\right|=\left|S_{n}(132)\right|=c_{n}=\frac{1}{n+1}\left(\begin{array}{c}2 n \\ n\end{array}\right)$ by using generating trees. The idea is to define a statistic on a permutation in $S_{n}(123)$ so that we can count the permutations in $S_{n+1}(123)$ by inserting $n+1$ into permutations in $S_{n}(123)$. For the purpose of enumeration, we may only keep track of the statistics of the permutations in the process of generating the permutations in $S_{n}(123)$ starting from the permutation $(1) \in S_{1}(123)$.

In general, to generate permutations in $S_{n}(\tau)$ we need the concepts of active and inactive sites. Given a permutation $\pi=\pi_{1} \pi_{2} \cdots \pi_{n} \in S_{n}(\tau)$, we say that the element $\pi_{i}$ is in site $i$. Moreover, a site $i$ is called an active site if the insertion of $n+1$ into the site $i$ produces a permutation $\pi^{\prime} \in S_{n+1}(\tau)$; otherwise the site $i$ is said to be inactive. West showed that the generating trees for 123-avoiding permutations and 132-avoiding permutations correspond to the same root label and the same succession rules:

$$
\left\{\begin{array}{l}
\text { Root : }(2) \\
\text { Rule }:(t) \rightsquigarrow(2)(3)(4) \ldots(t+1) .
\end{array}\right.
$$

One may check that for both $S_{n}(123)$ and $S_{n}(132)$ the label $(i)$ comes from the number of active sites of a permutation. For the above generating tree, the label of the root is (2); the labels at level two are (2)(3); the labels at level three are $(2)(3)(2)(3)(4)$; and the labels at level four are

$$
(2)(3)(2)(3)(4)(2)(3)(2)(3)(4)(2)(3)(4)(5) \text {. }
$$


It is not difficult to verify that the number of labels at level $n$ equals the Catalan number $c_{n}$.

We now consider matchings avoiding certain patterns by using the technique of generating trees. Given a matching $\pi$ on $[2 n]$, a position $s$ of $\pi$ is meant to be the position between the nodes $s$ and $s+1$ if $1 \leq s \leq 2 n-1$, and the position $2 n$ is meant to be the position to the right of the node $2 n$. For the purpose of generating matchings with $n+1$ edges from a matching with $n$ edges, we always consider the edge with the rightmost initial point. Let $\pi$ be a matching on $[2 n]$. We can obtain a matching $\pi^{\prime}$ on $[2 n+2]$ by adding an edge from position $s$ to position $t$ with $1 \leq s \leq t \leq 2 n$ of the matching $\pi$, such that the inserted edge has the rightmost initial point in $\pi^{\prime}$. Conversely, we can reach a unique matching with $n$ edges from a matching $\pi$ with $n+1$ edges by deleting the edge of $\pi$ with the rightmost initial point.

Definition 5.1 Let $\tau$ be a pattern on $[k]$ and $\pi$ be a $\tau$-avoiding matching on $[2 n]$. The position $s$ of $\pi$ is an active site if there exists a position $t, 1 \leq s \leq t \leq 2 n$, such that inserting an edge from position $s$ to position $t$ gives a $\tau$-avoiding matching on $[2 n+2]$, in which the inserted edge has the rightmost initial point. Otherwise, it is called an inactive site.

In the following structural lemma, we aim to characterize the generating tree $T(\tau)$ for any $\tau \in\{12312,12132,12123,12321,12231,12213\}$. We will recursively define a rooted tree $T(\tau)$ in which the vertices on the $n$-th level correspond to $\tau$-avoiding matchings with $n$ edges. We start with the matching with only one edge, and let $\pi \in \mathcal{M}_{n}(\tau)$ be a child of $\pi^{\prime} \in \mathcal{M}_{n-1}(\tau)$ obtained from $\pi$ by deleting the edge with the rightmost initial point. It will be convenient to label each vertex of the generating tree $T(\tau)$ with the number of active sites of the associated matching minus 2 . Let $\pi$ be a matching with $n$ edges and $k$ active sites, then we can get $\left(\begin{array}{c}k+1 \\ 2\end{array}\right)$ different matchings with $n+1$ edges by inserting an edge into any two of its active sites. Hence, in the generating tree $T(\tau)$, if a vertex has

label $(k)$, then it has $\left(\begin{array}{c}k+3 \\ 2\end{array}\right)$ children. As usual, we use $(k)^{s}$ to denote $s$ occurrences of a label $(k)$.

Lemma 5.2 For any $\tau \in\{12312,12132,12123,12321,12231,12213\}, T(\tau)$ can be characterized by the following succession rules:

$$
\left\{\begin{array}{l}
\text { Root : }(0) \\
\text { Rule }:(k) \rightsquigarrow(k+1)^{1}(k)^{2}(k-1)^{3} \ldots(0)^{k+2} .
\end{array}\right.
$$

Proof. We only consider three cases $\tau=12312, \tau=12123$ and $\tau=12321$. The other cases are similar.

The case $\tau=12312$ : The matching $\pi$ on [2] has two active sites which gives the root $(0)$. Let $\pi$ be a 12312 -avoiding matching on $[2 n]$ labeled by $(k)$ with active sites $i_{1}, i_{2}, \ldots, i_{k+2}$. 
Let $\pi^{\prime}$ be a matching in $\mathcal{M}_{n+1}$ (12312) obtained from $\pi$ by inserting an edge from position $i_{s}$ to position $i_{t}$ with $i_{s} \leq i_{t}$. Hence the active sites of $\pi^{\prime}$ are $i_{t}+1, i_{t}+2, i_{t+1}+2, \ldots, i_{k+2}+2$ so that there are $k+4-t$ active sites. Therefore, the children of a node $(k)$ are exactly the nodes $\left(k^{\prime}\right)$ where $k^{\prime}=k+2-t$. If $s$ ranges over $1,2, \ldots, k+2$ and $t$ ranges over $s, s+1, \ldots, k+2$, we get the rules 5.1 .

The case $\tau=12123$ : The matching $\pi$ on [2] has two active sites, which gives the root $(0)$. Let $\pi$ be a 12123 -avoiding matching on $[2 n]$ labeled by $(k)$ with active sites $i_{1}, i_{2}, \ldots, i_{k+2}$. Let $\pi^{\prime}$ be a matching in $\mathcal{M}_{n+1}(12123)$ obtained from $\pi$ by inserting an edge from position $i_{s}$ to position $i_{t}$. Then the active sites of $\pi^{\prime}$ are $i_{s}+1, i_{s+1}+1, \ldots, i_{t}+1$ when $i_{s} \neq i_{t}$ and $i_{s}+1, i_{s}+2, i_{s+1}+2, \ldots, i_{k+2}+2$ when $i_{s}=i_{t}$. Hence the label of $\pi^{\prime}$ is $\left(k^{\prime}\right)$ where $k^{\prime}=t-s-1$ if $i_{s} \neq i_{t}$ and $k^{\prime}=k-s+2$, otherwise. If $s$ ranges over $1,2, \ldots, k+1$ and $t$ ranges over $s+1, \ldots, k+2$ for the first case and $s$ ranges over $1,2, \ldots, k+2$ for the second case, we get the rules 5.1.

The case $\tau=12321$ : The matching $\pi$ on [2] has two active sites, which gives the root $(0)$. Let $\pi$ be a 12321-avoiding matching on [2n] labeled by $(k)$ with active sites $i_{1}, i_{2}, \ldots, i_{k+2}$. It is clear that $i_{k+2}=2 n$. Let $\pi^{\prime}$ be a matching obtained from $\pi$ by inserting an edge from site $i_{s}$ and to site $i_{t}$. Then the active sites of $\pi^{\prime}$ are $i_{t}+2, i_{t+1}+2, \ldots, i_{k+2}+2$ when $t<k+2$ and $i_{s}+1, i_{s+1}+1 \ldots, i_{k+2}+1, i_{k+2}+2$ when $t=k+2$. It follows that the label of $\pi^{\prime}$ is $\left(k^{\prime}\right)$ where $k^{\prime}=k-t+1$ when $t<k+2$ and $k^{\prime}=k+2-s$, otherwise. If $s$ ranges over $1,2, \ldots, k+1$ and $t$ ranges over $s, s+1, \ldots, k+1$ for the first case and $s$ ranges over $1,2, \ldots, k+2$ and $t=k+2$ for the second case, we get the rules 5.1.

Applying Theorem 2.3 and Lemma 5.2, we reach the following conclusion.

Theorem 5.3 For $\tau \in\{12312,12132,12123,12321,12231,12213\}$, we have $\left|M_{n}(\tau)\right|=$ $C_{n, 3}$.

Acknowledgments. The authors would like to thank the referee for helpful comments leading to an improvement of an earlier version. This work was supported by the 973 Project on Mathematical Mechanization, the National Science Foundation, the Ministry of Education, and the Ministry of Science and Technology of China.

\section{References}

[1] E. Barcucci, A. del Lungo, E. Pergola, R. Pinzani, ECO: a methodology for the enumeration of combinatorial objects, J. Differential Equations Appl. 5 (1999) 435490.

[2] A. Berele, A Schensted-type correspondence for the symplectic group, J. Combin. Theory, Ser. A 43 (1986), 320-328.

[3] L. Carlitz, Enumeration of two-line arrays, Fibonacci Quart. 11 (1973) 113-130. 
[4] W.Y.C. Chen, A general bijective algorithm for increasing trees, Systems Science and Mathematical Sciences 12 (1999) 194-203.

[5] W.Y.C. Chen, E.Y.P. Deng, R.R.X. Du, Reduction of $m$-regular noncrossing partitions, Europ. J. Combin. 26 (2005) 237-243.

[6] W.Y.C. Chen, E.Y.P. Deng, R.R.X. Du, R.P. Stanley, C.H.F. Yan, Crossings and nestings of matchings and partitions, Trans. Amer. Math. Soc., to appear.

[7] W.Y.C. Chen, S.H.F. Yan, Noncrossing trees and noncrossing graphs, Electron. J. Combin., 13 (2006) N12.

[8] F.R.K. Chung, R.L. Graham, V.E. Hoggat, M. Kleiman, The number of Baxter permutations, J. Combin. Theory, Ser. A 24 (1978) 382-394.

[9] H. Davenport, A. Schinzel, A combinatorial problem connected with differetial equations, Amer. J. Math. 87 (1965) 684-694.

[10] M. Delest, S. Dulucq, L. Favrean, An analogue to the Robinson-Schensted correspondence for oscillating tableaux, in: L. Cerlienco, D. Foata (Eds.), Séminaire Lotharingien de Combinatoire (Alghero, 1988), University of Louis Pasteur, Strasbourg 1988.

[11] E. Deutsch, Dyck path enumeration, Discrete Math. 204 (1999) 167-202.

[12] E. Deutsch, S. Feretic, M. Noy, Diagonally convex directed polyominoes and even trees: a bijection and related issues, Discrete Math. 256 (2002) 645-654.

[13] E. Deutsch, M. Noy, Statistics on non-crossing trees, Discrete Math. 254 (2002) $75-87$.

[14] S. Feretic, D. Svrtan, Combinatorics of diagonally convex directed polyominoes, Discrete Math. 157 (1996) 147-168.

[15] M. Noy, Enumeration of noncrossing trees on a circle, Discrete Math. 180 (1998) 301-313.

[16] I.M. Gessel, X.G. Viennot, Determinants, paths and plane partitions, preprint, 1989.

[17] I.P. Goulden, D.M. Jackson, Combinatorial Enumeration, John Wiley, New York, 1983.

[18] D. Gouyou-Beauchamps, Standard Young tableaux of height 4 and 5, Europ. J. Combin. 10 (1989) 69-82.

[19] D. Gouyou-Beauchamps, Chemins sous-diagonaux et tableau de Young, Combinatoire Enumerative (Montreal 1985), Lecture Notes in Math. 1234 (1986) 112-125.

[20] P. Hilton, J. Pedersen, Catalan numbers, their generalization, and their uses, Math. Intell. 13 (1991) 64-75.

[21] M. Klazar, On abab-free and abba-free set partitions, Europ. J. Combin. 17 (1996) 53-68.

[22] M. Klazar, Bell numbers, their relatives, and algebraic differential equations, J. Combin. Theory, Ser. A 102 (2003) 63-87. 
[23] M. Klazar, Non-P-recursiveness of numbers of matchings or linear chord diagrams with many crossings, Adv. Appl. Math. 30 (2003) 126-136.

[24] R.C. Mullin, R.G. Stanton, A map-theoretic approach to Davenport Schinzel sequences, Pacific J. Math. 40 (1972) 167-172.

[25] A. de Médicis, X.G. Viennot, Moments des $q$-polynômes de Laguerre et la bijection de Foata-Zeilberger, Adv. Appl. Math. 15 (1994) 262-304.

[26] A. Panholzer, H. Prodinger, Bijections for ternary trees and noncrossing trees, Discrete Math. 250 (2002) 181-195.

[27] T.W. Roby, Applications and Extenstions of Fomin's Generalization of the RobinsonSchensted Correspondence to Differential Posets, Ph.D. Thesis, Massachusetts Institue of Technology, 1991.

[28] M. de Sainte-Catherine, Couplages et Pfaffiens en Combinatoire, Physique et Informatique, Ph.D. Thesis, University of Bordeaux I, 1983.

[29] N.J.A. Sloane, The On-Line Encyclopedia of Integer Sequences, http://www.research.att.com/ njas/sequences.

[30] Z.E. Stankova, Forbidden subsequences, Discrete Math. 132 (1994) 291-361.

[31] Z.E. Stankova, Classification of forbidden subsequences of length 4, Europ. J. Combin. 6 (1985) 383-406.

[32] P.R. Stanley, Enumerative Combinatorics, Vol. 2, Cambridge University Press, 1999.

[33] P.R. Stein, On a class of linked diagrams, I. Enumerations, J. Combin. Theory, Ser. A 24 (1978) 357-366.

[34] S. Sundaram, On the Combinatorics of Represetations of $S p(2 n, \mathbb{C})$, Ph.D. Thesis, Massachusetts Institute of Technology, 1978.

[35] S. Sundaram, The Cauchy identity for Sp(2n), J. Combin. Theory, Ser. A 53 (1990) 209-238.

[36] J. Touchard, Sur une probléme de configurations et sur les fractions continues, Canad. J. Math. 4 (1952) 2-25.

[37] J. West, Generating trees and the Catalan and Schröder numbers, Discrete Math. 146 (1995) 247-262.

[38] J. West, Generating trees and forbidden subsequences, Discrete Math. 157 (1996) $363-374$.

[39] H.S. Wilf, generatingfunctionology, Academic Press, 1994. 